\title{
Dislocation mobility in two-dimensional Lennard-Jones material
}

\author{
N.P. Bailey ${ }^{a, *}$, J.P. Sethna ${ }^{a}$, C.R. Myers ${ }^{b}$ \\ a Physics Department, Cornell University, 117 Clark Hall, Ithaca, NY 14853, USA \\ ${ }^{\mathrm{b}}$ Cornell Theory Center, Cornell University, Ithaca, NY 14853, USA
}

\begin{abstract}
In seeking to understand at a microscopic level the response of dislocations to stress, we have undertaken to study as completely as possible the simplest case: a single dislocation in a two-dimensional crystal. The intention is that results from this study will be used as input parameters in larger length scale simulations involving many defects. We present atomistic simulations of defect motion in a two-dimensional material consisting of atoms interacting through a modified Lennard-Jones potential. We focus on the regime where the shear stress is smaller than its critical value, where there is a finite energy barrier for the dislocation to hop one lattice spacing. In this regime motion of the dislocation will occur as single hops through thermal activation over the barrier. Accurate knowledge of the barrier height is crucial for obtaining rates of such processes. We have calculated the energy barrier as a function of two components of the stress tensor in a small system, and have obtained good fits to a functional form with only a few adjustable parameters. We examine prefactors, finite temperature and dynamics. (C) 2001 Elsevier Science B.V. All rights reserved.
\end{abstract}

Keywords: Dislocation mobility; Lennard-Jones; Energy barriers; Nudged elastic band

\section{Introduction}

In the interest of generating information from atomistic simulations for use in larger length-scale simulations, we have studied the motion of a single dislocation as a function of stress and temperature. We have worked in two dimensions (2D) to ease the computational effort and to aid visualization. We feel that, we have a chance of understanding this simplest dislocation problem in detail. Then it will make sense to proceed to more realistic, though computationally more expensive, cases (e.g. three dimensions, realistic potentials, etc.).

Our model consists of a two-dimensional crystal with a dislocation with Burgers vector in the vertical direction, and rigid walls on the sides with which a shear stress may be applied. The applied shear stress is the resolved shear stress in our geometry. At zero temperature, this causes the dislocation to glide if it is above a certain critical value $\sigma_{\mathrm{c}}$. Note that the critical resolved shear stress for dislocation motion depends on the other components of stress, hence knowledge of the resolved shear stress alone is not enough to decide, whether a given dislocation will move or not. At zero temperature, with $\sigma_{x y}<\sigma_{\mathrm{c}}$, the dislocation cannot move, but with a finite temperature, motion still occurs as thermally activated hops over an energy barrier. This barrier

\footnotetext{
${ }^{*}$ Corresponding author.

E-mail address: nbailey@tristan.th.cornell.edu (N.P. Bailey).
}

corresponds to the Peierls barrier for an edge dislocation in three dimensions. Our task was to calculate this barrier as a function of all three components of the stress tensor $\left(\sigma_{x x}\right.$, $\left.\sigma_{x y}, \sigma_{y y}\right)$. However, difficulties with $\sigma_{y y}$ have led us to start considering different kinds of boundary conditions. We have considered only one size of system; finite size effects are important - this also relates to the boundary conditions. To check the validity of our approach, we simulated the system under stress at several temperatures and measured the velocity of the dislocation of a certain time length. We obtain mostly good agreement between the rate predicted by transition state theory and the measured velocities, see Fig. 1.

\section{Computation and simulation}

For values of shear less than the critical shear stress, there exist so called fixed points of the dynamics, i.e. states which do not change with time (at zero temperature). These are associated with local minima in the potential. Two nearby minima are separated by a barrier in the energy landscape (the saddle point of the barrier is also a fixed point, albeit an unstable one). At nonzero temperature but which is low compared to the barrier, the transition rate between the states will have the form

$R=v \exp \left(-\frac{E_{\mathrm{B}}}{k_{\mathrm{B}} T}\right)$ 


\section{Log of Hopping Rate vs Inverse Temperature}

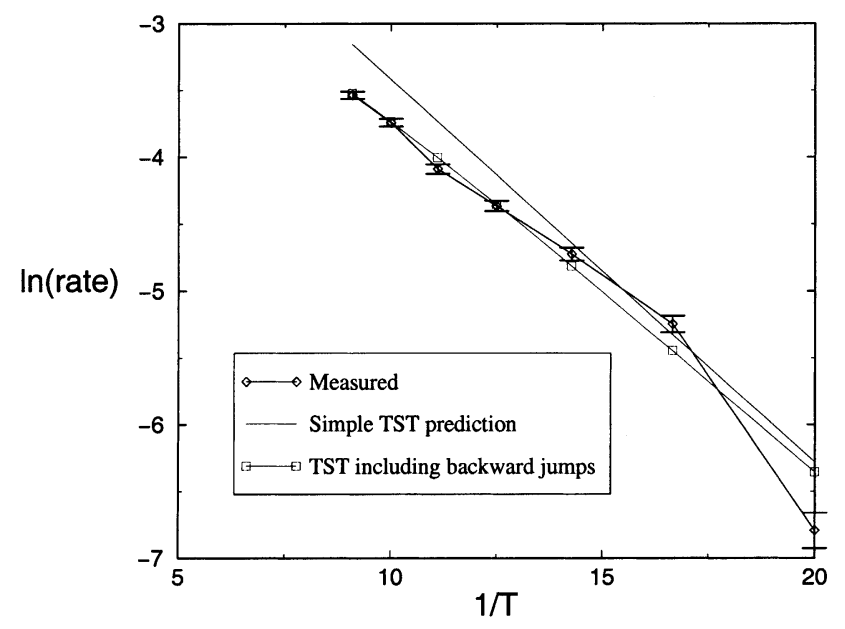

Fig. 1. Arrhenius plot of rate (number of hops per unit time). Notice that including the contribution from backward jumps makes a significant improvement.

where $E_{\mathrm{B}}$ is the barrier height and $v$ an attempt frequency which can be calculated from the curvature of the potential landscape near the minimum and near the barrier top [5]. Because of the exponential, however, the rate is much more sensitive to $E_{\mathrm{B}}$ than it is to $v$. Hence, it is important to know $E_{\mathrm{B}}$ accurately to calculate such rates.

The system consists of a relatively small $(<100)$ number of atoms in 2D interacting through a classical pair potential. The lattice is triangular with one basis vector in the vertical $(y)$ direction. A dislocation with Burger's vector in the $y$-direction is introduced by applying the formula for the displacement field of an edge dislocation to the positions of all the atoms, and then removing atoms which overlap. The system has periodic boundary conditions in the $y$-direction, and rigid 'walls' on the sides. The walls are simply lines of atoms (corresponding to crystal columns, given the orientation of the crystal) which are constrained to move as rigid bodies. This allows a well-defined external force to be applied to each boundary. If a shear stress is applied to the boundary walls, namely, an upward force on one and a downward force on the other, the dislocation will move upward or downward by glide, since the Burger's vector and hence the glide direction are in the $y$-direction and such an applied shear stress is equal to the resolved shear stress for this geometry. In addition to the hard outer walls, the next-to-outermost columns of atoms are constrained to always have the same $x$-coordinate. We find that softening the boundaries with these extra 'semi-rigid' columns cuts down reflections from the boundaries when running dynamical simulations. Apart from these outer two columns on each side, the system is square. We use a classical pair potential defined as follows: Lennard-Jones (six to twelve, with standard parameters $\epsilon$ and $\sigma$ ) for $r<r_{\text {cut } 1}=2.41308788 \sigma$, a quadratic in $r^{2}$ for $r_{\text {cut } 1}<r<r_{\text {cut2 }}=2.7 \sigma$, and zero for $r>r_{\text {cut2 }}$. This cutoff is large enough for third neighbor interactions to be included. The potential was formulated by Chen [3]. It is continuous and smooth everywhere. The units used throughout the paper are those defined by the parameters $\epsilon$ and $\sigma$, and the atomic mass $m$, e.g. the unit of stress is $\epsilon / \sigma^{2}$.

The main part of the computation consisted of looping through ranges of stress value. The system is relaxed to find a minimum, then a procedure which generates the configuration corresponding to the dislocation having glided one lattice spacing is applied. Then these two configurations are passed to the barrier finding routine, which uses the Nudged elastic band method [1]. A chain of replicas of the system is created forming a line in configuration space between the local minima. The whole chain relaxed in such a way that it ends up along the minimum energy path. Calculating the actual barrier simply involves taking the four highest points in the energy versus distance along-the-path curve and fitting them to a cubic (a quadratic is not good enough when the barrier goes to zero). The difference between this result and that obtained by fitting to a quartic gives a handy error estimate. Note that by energy $(I)$ mean the total potential energy of the system including that associated with the applied stress. Then, the stress is incremented and the loop repeats with the next stress value.

To check that the rate was correctly given by the barrier, we simulated the system at finite temperature using Langevin dynamics. The dislocation velocity can be found by tracking the boundary walls. For sufficiently low temperature, only one process is relevant: single hops in the direction of the applied stress. However, we find better agreement between the predicted rate and the rate measured by simulation when we take into account the next most important process, backward jumps. This is more the case at higher temperatures.

\section{Results}

The energy barrier as a function of shear stress $\sigma_{x y}$ with fixed $\sigma_{x x}$ for several values $\sigma_{x x}$ of is shown in Fig. 2. The dots are data points from barrier calculations; the solid lines are three-parameter fits to a series expansion obtained by considering the one-dimensional barrier problem (see below). For stress larger than the critical value, there is no fixed point, and the defect slides with periodically varying velocity. For stress smaller than the critical value, there are two fixed points, a stable one corresponding to the local minimum, and an unstable one corresponding to the barrier top. Of course there are many more really, due to the periodicity of the lattice, but we focus on one particular local minimum, and the barrier between it and the next one. The appearance of two fixed points as the stress goes below $\sigma_{\mathrm{c}}$ (or equivalently their disappearance as stress goes above $\sigma_{\mathrm{c}}$ ) is a saddle-node bifurcation. Note that, we also have points for negative shear; this corresponds to hopping in the opposite direction for positive shear. For large negative shear the 


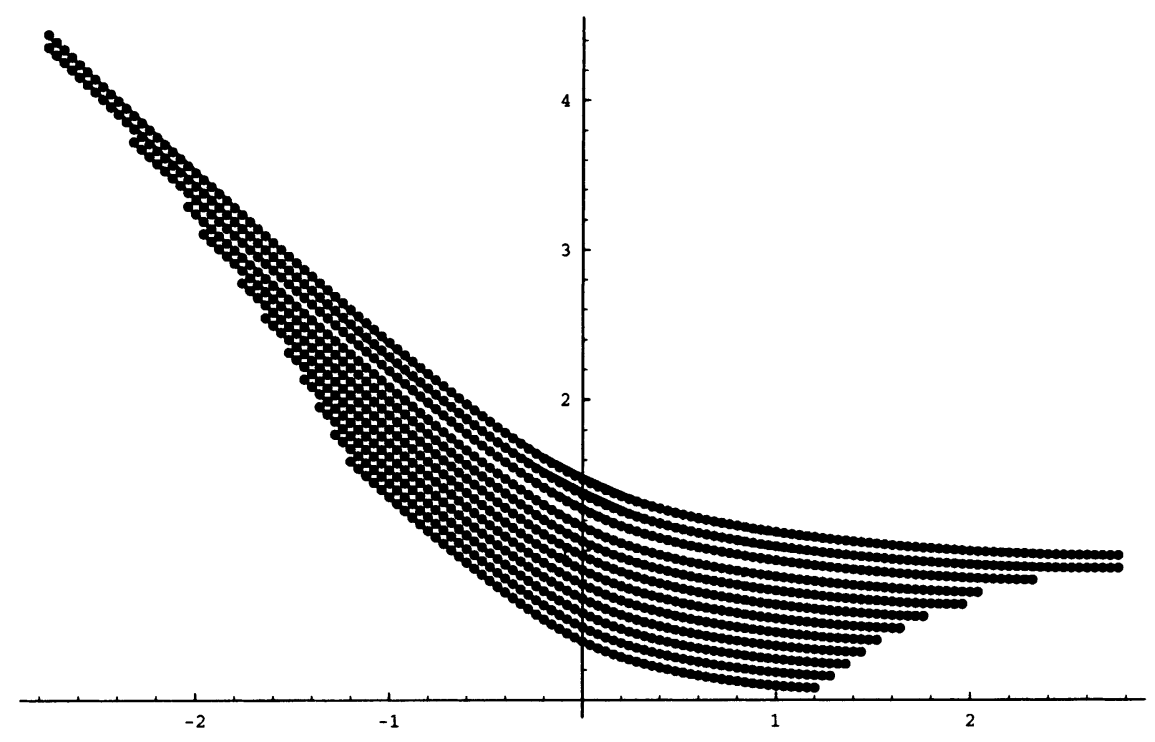

Fig. 2. Energy barrier vs. shear stress for $8.8<\sigma_{x x}<13.6$. The curves have been displaced vertically by 0.08 for clarity (the bottom curve is for smallest $\left.\sigma_{x x}\right)$. Also included, but not readily visible are the one-dimensional fits to the data.

barrier becomes simply the energy difference between the two minima. These local minima only exist for $\left|\sigma_{x y}\right|<\sigma_{\mathrm{c}}$ (beyond which the dislocation starts to slide in the appropriate direction), hence our data covers the range $-\sigma_{\mathrm{c}}$ to $\sigma_{\mathrm{c}}$ for several values of $\sigma_{x x}$.

\subsection{Functional forms: insight from $1 D$}

We can gain considerable insight just by thinking about a barrier-type problem for a single degree of freedom (1D). Dynamical systems theory, in particular the theory of normal forms [4] justifies this kind of thinking. Consider the normal form of a saddle-node bifurcation in a one-dimensional problem (see e.g. [2])

$\dot{X}=-\epsilon+X^{2}$

here $\epsilon$ is the control parameter which vanishes at the bifurcation, corresponding to $\sigma_{\mathrm{c}}-\sigma_{x y}$ in our case. This should be considered the equation of motion of a system which is over-damped. The right hand side corresponds to the force, which can be derived from the potential

$V(x)=\epsilon X-\frac{1}{3} X^{3}$

The energy barrier is the difference between the potential at the maximum and the minimum, and it is $(4 / 3) \epsilon^{3 / 2}$. In general, with more terms added to Eq. (3), for example $x r$ and $r^{2}$ terms, one obtains a series expansion of the barrier height in $\epsilon$, containing half-integer powers of $\epsilon$ starting with $\epsilon^{3 / 2}$. This suggests fitting of our data to a function like $f(x)=\alpha\left(\sigma_{\mathrm{c}}-\sigma_{x y}\right)^{1.5}+\beta\left(\sigma_{\mathrm{c}}-\sigma_{x y}\right)^{2.5}$. However, it is possible to do better by taking into account other information, namely the symmetry between positive and negative shear. The difference $E_{\mathrm{B}}\left(-\sigma_{x y}\right)-E_{\mathrm{B}}\left(\sigma_{x y}\right)$ is simply the difference in the heights of the forward and backward barriers. Now, because of the periodicity of the system, the atomic configurations at these two points are identical (up to permutations of the atoms), hence the only difference in energy must be due to the external forces. It is equal to the force times the displacement of the coordinate coupled to the force. We have equal and opposite forces on the boundaries; the work is their relative displacement multiplied by the force. To find the displacement for one hop, note that $N$ hops displace the boundaries by $\left(L_{y} /(N-1)\right)$, so for one hop it is $\left(L_{y} / N(N-1)\right)$. For the fixed $L_{y}$ simulations, we know $L_{y}$ precisely. The force is $\sigma_{x y} L_{y}$, so we have that

$E_{\mathrm{B}}\left(-\sigma_{x y}\right)-E_{\mathrm{B}}\left(\sigma_{x y}\right)=\frac{\sigma_{x y} L_{y}^{2}}{N(n-1)}$

which implies that $E_{\mathrm{B}}\left(\sigma_{x y}\right)+\left(\sigma_{x y} L_{y}^{2} / 2 N(N-1)\right)=$ $\left.E_{\mathrm{B}}\left(\sigma_{x y}\right)+\kappa \sigma_{x y}\right)$ is an even function of $\sigma_{x y}$, say $g\left(\sigma_{x y}\right)$. We now guess the form

$g\left(\sigma_{x y}\right)=\sum_{n=0,2,3,4, \ldots} a_{n}\left(\sigma_{\mathrm{c}}^{2}-\sigma_{x y}^{2}\right)^{n / 2}$

This is required to satisfy certain conditions. First, its slope at $\sigma_{\mathrm{c}}$ must be $\kappa$ so that $E_{\mathrm{B}}$ has no slope there. Next, when on expands in $\sigma_{\mathrm{c}}-\sigma_{x y}$, only the odd half integer terms should appear, starting from 3/2. Mathematics was used to compute the coefficients of the even $n$-terms in terms of the other coefficients and $\sigma_{\mathrm{c}}$. We truncated the series (5) after $n=6$ which leaves three adjustable parameters: $a_{3}, a_{5}$ and $\sigma_{\mathrm{c}}$. A separate nonlinear least squares fit was performed for each subset of the data corresponding to a particular $\sigma_{x x}$. The parameters are regular functions of $\sigma_{x x}$, and were fitted to simple Taylor series up to quadratic order. The results from these fits were used to supply initial guesses for a single fit to the entire data 


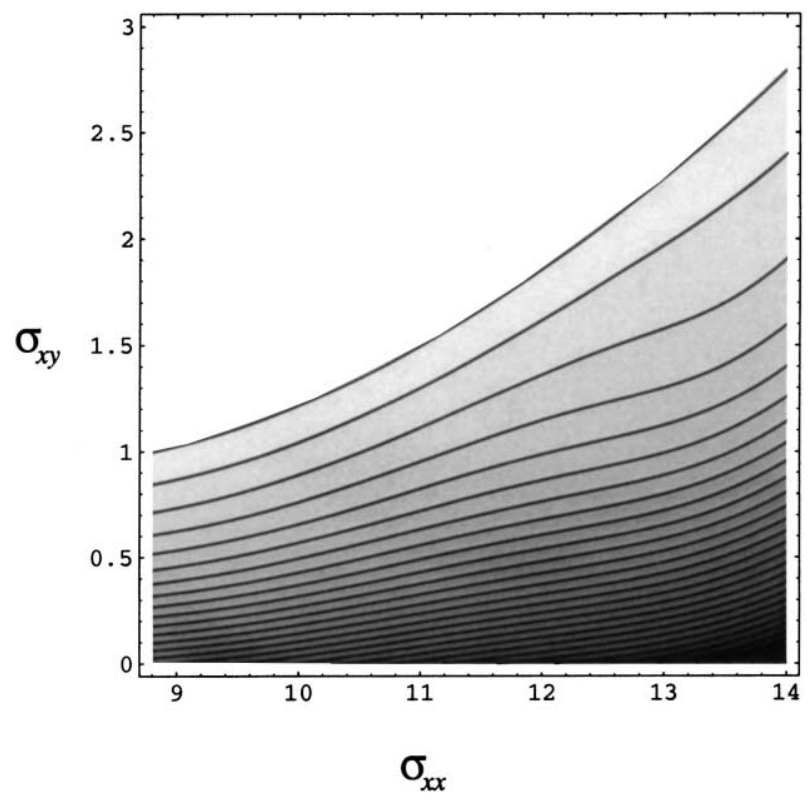

Fig. 3. Contour plot of the entire data set fitted in both $\sigma_{x x}$ and $\sigma_{x y}$. The top line corresponds to $E_{\mathrm{B}}=0.0$, and hence give $\sigma_{\mathrm{c}}$ as a function of $\sigma_{x x}$.

set as a function of both $\sigma_{x y}$ and $\sigma_{x x}$. This fit had nine parameters. Fig. 3 shows a contour plot of the resulting function of $\sigma_{x x}$ and $\sigma_{x y}$. The top contour is $\sigma_{\mathrm{c}}$ as a function of $\sigma_{x x}$. The error between the fit values and the original data varies, but is generally about $1 \%$. The estimated errors on the individual barrier calculations is smaller than this, around $0.1 \%$.

\section{Conclusions and future work}

We have calculated the barrier height for dislocation hopping for range of both $\sigma_{x x}$ and $\sigma_{x y}$. We have shown, how this data can be parametrized to reasonable accuracy with a fit that needs only a few parameters. The present results, augmented by $\sigma_{y y}$ dependence, could be used to calculate the motion of dislocations in a dislocation dynamics simulation.

Attempts to include the dependence on the third component of the stress tensor, $\sigma_{y y}$, which corresponds to pressure on the top and bottom of the system lead to the realization that our rectangular periodic boundary conditions cause unphysical stresses in the system. This leads to a strong sensitivity of the barrier heights to small changes in the length of the simulation box for a fixed number of atoms, as well to changing the overall size of the system (e.g. going from $7 \times 7$ to $9 \times 9$ ). We are planning to repeat our work using a system with boundary conditions given by continuum theory. Eventually, we would like to do similar work in three dimensions with realistic potentials.

\section{Acknowledgements}

This project was supported by NSF Grant No. DMR 9873214, and was done using the Intel/NT Velocity Cluster at the Cornell Theory Center. We had helpful discussions with Tejs Vegge, Enrique Batista and Markus Rauscher.

\section{References}

[1] T. Rasmussen, K.W. Jacobsen, T. Leffers, O.B. Pedersen, S.G. Srinivasan, H. Jonsson, Phys. Rev. Lett. 79 (1997) 3676.

[2] S.H. Strogatz, Nonlinear Dynamics and Chaos, Addison-Wesley, Reading, MA, 1994, p. 49.

[3] X. Chen, private communication.

[4] J. Guckenheimer, P. Holmes, Nonlinear Oscillations, Dynamical Systems, and Bifurcations of Vector Fields, Springer, Berlin, 1983, p. $138 \mathrm{ff}$.

[5] P. Hänggi, P. Talkner, M. Borkovec, Rev. Mod. Phys. 62 (1990) 251. 